Review

\title{
SRSF3: Newly discovered functions and roles in human health and diseases
}

\author{
Dhanashree Anil More, Arun Kumar* \\ Department of Molecular Reproduction, Development and Genetics, Indian Institute of Science, Bangalore, 560012, India
}

\section{A R T I C L E I N F O}

\section{Keywords:}

Splicing factors

SR proteins

SRSF

SRSF3

Cancer

Disease

\begin{abstract}
A B S T R A C T
The serine/arginine rich proteins (SR proteins) are members of a family of RNA binding proteins involved in regulating various features of RNA metabolism, including pre-mRNA constitutive and alternative splicing. In humans, a total of 12 SR splicing factors (SRSFs) namely SRSF1-SRSF12 have been reported. SRSF3, the smallest member of the SR family and the focus of this review, regulates critical steps in mRNA metabolism and has been shown to have mRNA-independent functions as well. Recent studies on SRSF3 have uncovered its role in a wide array of complex biological processes. We have also reviewed the involvement of SRSF3 in disease conditions like cancer, ageing, neurological and cardiac disorders. Finally, we have discussed in detail the autoregulation of SRSF3 and its implications in cancer and commented on the potential of SRSF3 as a therapeutic target, especially in the context of cancer.
\end{abstract}

\section{Introduction}

The serine/arginine rich proteins (SR proteins) belong to a family of phylogenetically conserved RNA binding proteins found in all metazoans and plants. SF2 (SRSF1) was the first human SR protein identified by complementation of cytoplasmic S-100 extract from HeLa cells that is deficient in splicing (Krainer et al., 1990). Zahler et al. (1992) first referred to SR proteins as set of proteins containing consecutive serine ( $\mathrm{S}$ ) and arginine (R) dipeptides sequences and classified them as a family based on the presence of phosphoepitope recognized by a monoclonal antibody, their conservation across animal kingdom and their ability to complement the splicing-deficient cytoplasmic S-100 extract. The new nomenclature proposed by Manley and Krainer (2010) defines SR proteins as "any protein with one or two N-terminal RNAbinding domains (RBDs; also called as RNA recognition motifs [RRMs]), followed by a downstream RS domain of at least 50 amino acids with $>40 \%$ RS content, characterized by consecutive RS or SR repeats". According to these rigorous criteria, humans contain 12 SR proteins designated as "SRSF" (serine/arginine rich splicing factor) followed by the numbers 1-12 (see Fig. 1; Table 1), reflecting the sequential order in which the proteins/genes were discovered. During constitutive and alternative splicing, the RRM domain imparts substrate specificity, while the RS domain is involved in protein-protein interactions. The zinc knuckle, a CCHC-type zinc finger (Fig. 1) is a unique feature of SRSF7 and contributes to the specificity of its RRM domain (Cavaloc et al., 1999).

SR proteins are localized in the nucleus and are distributed both in the nucleoplasm and in interchromatin granule clusters (IGCs) or nuclear speckles at steady state (Spector and Lamond, 2011). However, a subset of SR proteins can shuttle between the nucleus and the cytoplasm. Using domain swapped chimeric proteins, Ca'ceres et al. (1998) have demonstrated that the RS domain along with the RRM domain plays an important role in nucleocytoplasmic shuttling of these proteins. Phosphorylation of the RS domain is critical for both the nucleocytoplasmic shuttling and localization of SR proteins to the nuclear speckles (Ca'ceres et al., 1998; Lai et al., 2001; Koizumi et al., 1999). While SRSF1, SRSF3, SRSF7 and SRSF10 have rapid shuttling rates between the nucleus and cytoplasm, SRSF4 and SRSF6 shuttle at slower dynamic rates (Ca'ceres et al., 1998; Cowper et al., 2001; Cazalla et al., 2002; Sapra et al., 2009).

SR proteins are phosphorylated predominantly within the RS domain by two families of protein kinases, namely SR-specific protein kinases (SRPKs) and Cdc2-like kinases (CLKs), and the phosphorylation is crucial for both splicing and splicing-independent functions of SR proteins (Zhou and Fu, 2013; Aubol et al., 2013). SRPKs localize mainly to the cytoplasm, while CLKs are predominantly localized to the nucleus and each phosphorylate SR proteins by distinct mechanisms (Aubol et al., 2013; Long et al., 2019). Colwill et al. (1996) have reported that CLKs also play a critical role in regulating the subnuclear distribution of SR proteins. Corkery et al. (2015) have reviewed the implications of dysregulation of splicing kinases in tumorigenesis and therapeutic response to chemotherapy and radiation, further underscoring the importance of splicing kinases in regulation of SR proteins.

Here we review the regulation and recently identified functions of

\footnotetext{
* Corresponding author.

E-mail address: arunk@iisc.ac.in (A. Kumar).
} 


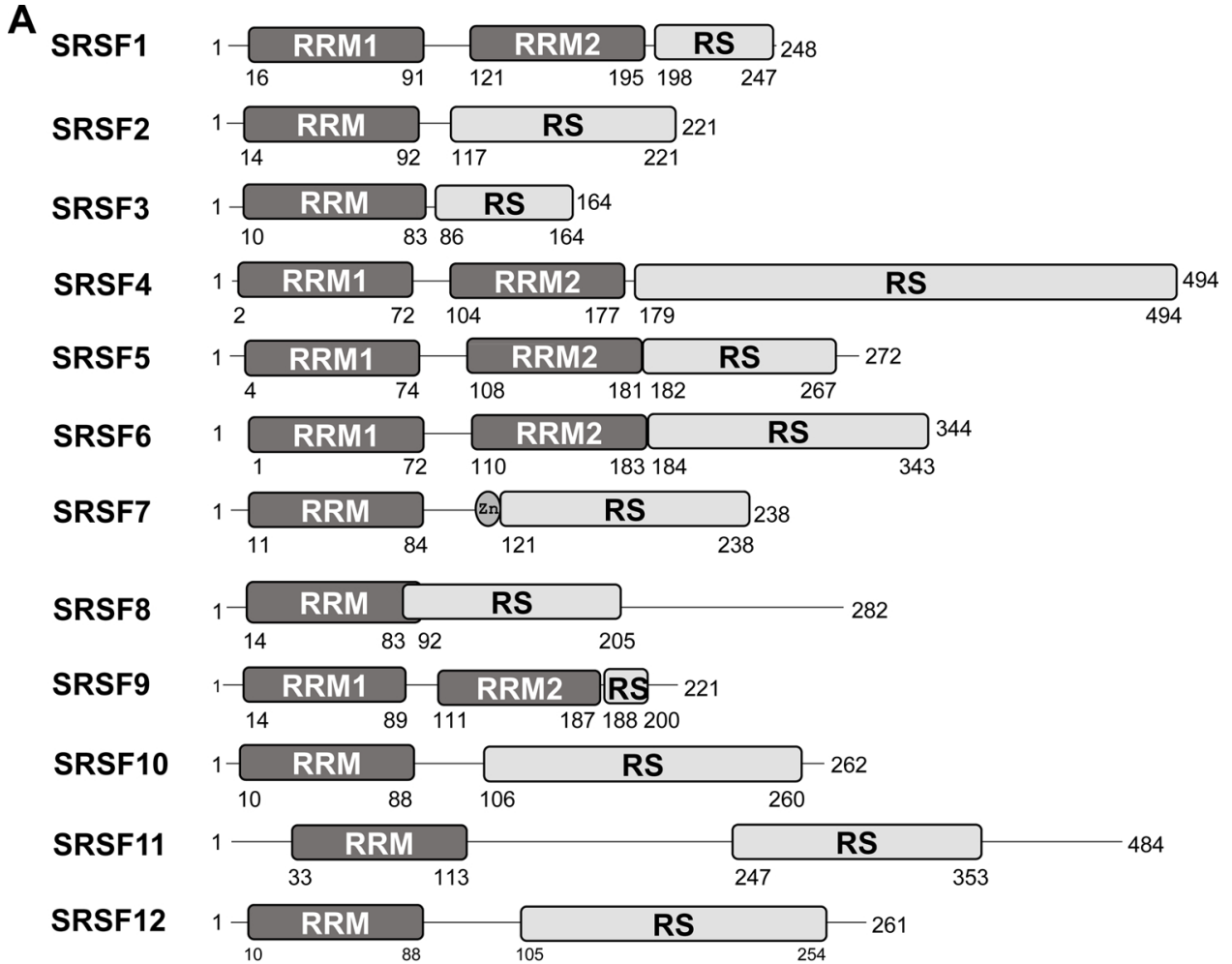

Fig. 1. Schematic representation of domain structure of SR proteins. Panel A: SR proteins have one or two N-terminal RRMs and Cterminal RS domain. The numbers refer to amino acid positions. Panel $B$ : Primary structure of the SRSF3 protein, highlighting the Nterminal RRM and C-terminal RS domain. Information regarding SRSF proteins was obtained from EMBL-EBI, NCBI and UniProt. Abbreviations: RRM, RNA recognition motif; and RS, Serine/arginine-rich domain; Zn, Zn knuckle-CCHC-type Zinc finger.

B

MHRDSCPLDCKVYVGNLGNNGNKTELERAFGYYGPLRSVWVARNPPGFAFVEFEDPRDAA

DAVRELDGRTLCGCRVRVELSNGEKRSRNRGPPPSWGRRPRDDYRRRSPPPRRRSPRRRS FSRSRSRSLSRDRRRERSLSRERNHKPSRSFSRSRSRSRSNERK

Table 1

Human SR proteins and genes.

\begin{tabular}{|c|c|c|c|c|}
\hline $\begin{array}{l}\text { Protein/Gene } \\
\text { symbol }\end{array}$ & $\begin{array}{l}\text { Chromosome } \\
\text { location }\end{array}$ & Aliases & GeneID & $\begin{array}{l}\text { UniProtKB } \\
\text { identifiers }\end{array}$ \\
\hline SRSF1/SRSF1 & $17 q 22$ & $\begin{array}{l}\text { ASF, MGC5228, SF2, } \\
\text { SF2p33, SFRS1, SRp30a }\end{array}$ & 6426 & Q07955 \\
\hline SRSF2/ SRSF2 & $17 q 25.1$ & $\begin{array}{l}\text { PR264, SC-35, SC35, } \\
\text { SFRS2, SFRS2A, SRp30b }\end{array}$ & 6427 & Q01130 \\
\hline SRSF3/ SRSF3 & $6 \mathrm{p} 21.31$ & SFRS3, SRp20 & 6428 & P84103 \\
\hline SRSF4/ SRSF4 & 1p35.3 & SFRS4, SRP75 & 6429 & Q08170 \\
\hline SRSF5/ SRSF5 & $14 q 24.1$ & HRS, SFRS5, SRP40 & 6430 & Q13243 \\
\hline SRSF6/ SRSF6 & 20q13.11 & $\begin{array}{l}\text { B52, SFRS6, SRP55, } \\
\text { HEL-S-91 }\end{array}$ & 6431 & Q13247 \\
\hline SRSF7/ SRSF7 & $2 \mathrm{p} 22.1$ & 9G8, AAG3, SFRS7 & 6432 & Q16629 \\
\hline SRSF8/ SRSF8 & $11 q 21$ & SFRS2B, SRP46, DSM-1 & 10929 & Q9BRL6 \\
\hline SRSF9/ SRSF9 & $12 q 24.31$ & SFRS9, SRp30c & 8683 & Q13242 \\
\hline SRS10/ SRSF10 & 1p36.11 & $\begin{array}{l}\text { FUSIP1, FUSIP2, NSSR, } \\
\text { PPP1R149, SFRS13, } \\
\text { SFRS13A, SRp38, } \\
\text { SRrp40, TASR, TASR1, } \\
\text { TASR2 }\end{array}$ & 10772 & O75494 \\
\hline SRS11/ SRSF11 & $1 \mathrm{p} 31.1$ & $\begin{array}{l}\text { NET2, SFRS11, p54, } \\
\text { dJ677H15.2 }\end{array}$ & 9295 & Q05519 \\
\hline SRS12/ SRSF12 & 6q15 & $\begin{array}{l}\text { SFRS13B, SFRS19, } \\
\text { SRrp35 }\end{array}$ & 135295 & Q8WXF0 \\
\hline
\end{tabular}

SRSF3 and its implications in human health and diseases.

\section{SRSF3: smallest SR protein}

SRSF3, the smallest of the SR proteins, was first identified and cloned by Zahler et al. (1992). It plays an essential role in early vertebrate development as SRSF3-null mouse embryos fail to form blastocysts and die at the morula stage (Jumaa et al., 1999). The SRSF3 gene, located on chromosome $6 \mathrm{p} 21.31$, contains 6 exons and spans 10,155 base pairs on the plus $(+)$ strand. It encodes for two transcript variants which result from alternative splicing. The transcript variant-1 is the protein-coding variant, while the transcript variant- 2 is generated by inclusion of an alternatively spliced exon 4 , and contains a premature stop codon, making it a candidate for non-sense mediated decay (NMD). However, Jumaa and Nielsen (1997) have shown that the transcript variant-2 encodes for a truncated protein which lacks the Cterminus RS domain and plays a crucial role in autoregulation of SRSF3 (discussed later). The SRSF3 protein encoded by transcript variant-1 is a 164 amino acid protein with a molecular weight of about $19 \mathrm{kDa}$. The modular structure of SRSF3 protein consists of two domains, namely the $\mathrm{RRM}$ at the $\mathrm{N}$-terminus and the RS at the C-terminus (Fig. 1). The RS domain is necessary and sufficient in targeting SRSF3 to the nuclear speckles (Cá ceres et al., 1997). During alternative splicing, SRSR3 recognizes the CUC(U/G)UCY splicing enhancer sequence (Schaal and Maniatis, 1999). Xiao et al. (2016) recently showed that during splicing, the $\mathrm{N}^{6}$ - methyladenosine $\left(\mathrm{m}^{6} \mathrm{~A}\right)$ reader protein $\mathrm{YTH}$ Domain Containing 1 (YTHDC1) promotes exon inclusion of target mRNAs through facilitating SRSF3 while preventing SRSF10 mRNA binding. Alternative splicing by SRSF3 was also found to be regulated by the Cterminal domain of RNA pol II (Mata and Kornblihtt, 2006). Splicing events are generally tightly regulated, and any aberrations in the splicing machinery can have far reaching consequences for the cell. Table 2 contains a list of SRSF3-regulated alternative splicing targets which are altered in various human diseases.

\section{Roles of SRSF3 in various cellular processes}

SRSF3 is a multifunctional protein and participates in regulation of several cellular processes (Fig. 2). Apart from its canonical role in both 
constitutive and alternative splicing, SRSF3 also regulates additional aspects of RNA metabolism like alternative polyadenylation, mRNA export, transcription termination and miRNA biogenesis. These functions along with some newly identified roles in various cellular processes are the focus of this review.

\section{Role in alternative polyadenylation}

Alternative polyadenylation (APA) is one of the major mechanisms of gene regulation found across eukaryotes which leads to generation of distinct 3' ends on mRNAs and other RNA pol II transcripts through differential use of multiple poly(A) sites present mostly in the 3' untranslated regions (3'UTRs) of mRNAs (Tian and Manley, 2017). Apart from its general role in gene regulation, APA also regulates various cellular processes, including mRNA metabolism, protein diversity and protein localization (Tian and Manley, 2017). Derti et al. (2012) showed that around $70 \%$ of identified human genes have multiple poly (A) sites in their 3'UTRs and can express APA isoforms. APA is critical for proliferation and differentiation and dynamic changes in APA occur during various biological processes, including embryonic development, cell cycle and stem cell self-renewal (Ji et al., 2009; Park et al., 2016; Lackford et al., 2014). Alterations in APA due to loss or gain of individual poly(A) sites and global changes in poly(A) site usage have also been implicated in various human neurological, immunological and haematological diseases, as well as in cancer and reviewed recently by Gruber and Zavolan (2019).

APA is a tightly regulated process under the control of various cis elements, trans-acting factors, as well as transcription (Derti et al., 2012; Tian and Manley, 2017). Lou et al. (1998) first reported the role of SRSF3 along with U1 snRNPs in the regulation of alternative polyadenylation. A study by Müller-McNicoll et al. (2016) further strengthened the role of SRSF3 in regulating APA. They observed that SRSF3 knockdown leads to decrease in the abundance of long 3'UTR isoforms while increasing shorter 3'UTR isoforms. They additionally showed that SRSF3 works antagonistically to SRSF7 to regulate 3'UTR length and through interaction with Nuclear Transcription Factor, XBox Binding 1 (NFX1), links the alternative 3' end formation to mRNA export (Müller-McNicoll et al., 2016). Kasowitz et al. (2018) observed that YTHDC1 is crucial during mouse embryogenesis and germline development as it regulates APA of pre-mRNA transcripts in oocytes. YTHDC1 can interact with both SRSF3 and SRSF7 and the observed alterations in alternative polyadenylation in YTHDC1-deficient oocytes could be through this interaction (Kasowitz et al., 2018)

\section{Role in mRNA export}

The mRNA export pathway involves the active transport of mRNAs from the nucleus to the cytoplasm and is essential for gene expression in eukaryotes. The mRNA export machinery comprises of three classes of factors, namely the adaptor proteins which bind directly to the RNA, receptor proteins (NFX1/TAP) that recognize the adaptor proteins and the nuclear pore complex (NPC) (Cullen, 2000). The role of SRSF3 along with 9G8/SRSF7 as adaptor proteins in mRNA export was first studied by Huang and Steitz (2001). They showed that SRSF3 and 9G8/ SRSF7 synergistically interact with a 22-nt RNA element to promote intronless mRNA export and speculated a more general role for these proteins in mRNA export (Huang and Steitz, 2001). Hautbergue et al. (2008) demonstrated that SRSF3 and SRSF7 stimulate the nuclear export of mRNA by directly handing over the mRNA to NFX1/TAP during export. Recently, Müller-McNicoll et al. (2016) further investigated SRSF1-7 proteins for their potential as NFX1/TAP adaptors that couple pre-mRNA processing to mRNA export. They demonstrated that SRSF3 is the most potent NFX1/TAP adaptor and confers sequence specificity to NFX1/TAP binding. SRSF3 and SRSF7 were both shown to promote NFX1/TAP recruitment to the mRNA and couple alternative RNA processing to mRNA export (Müller-McNicoll et al., 2016). 


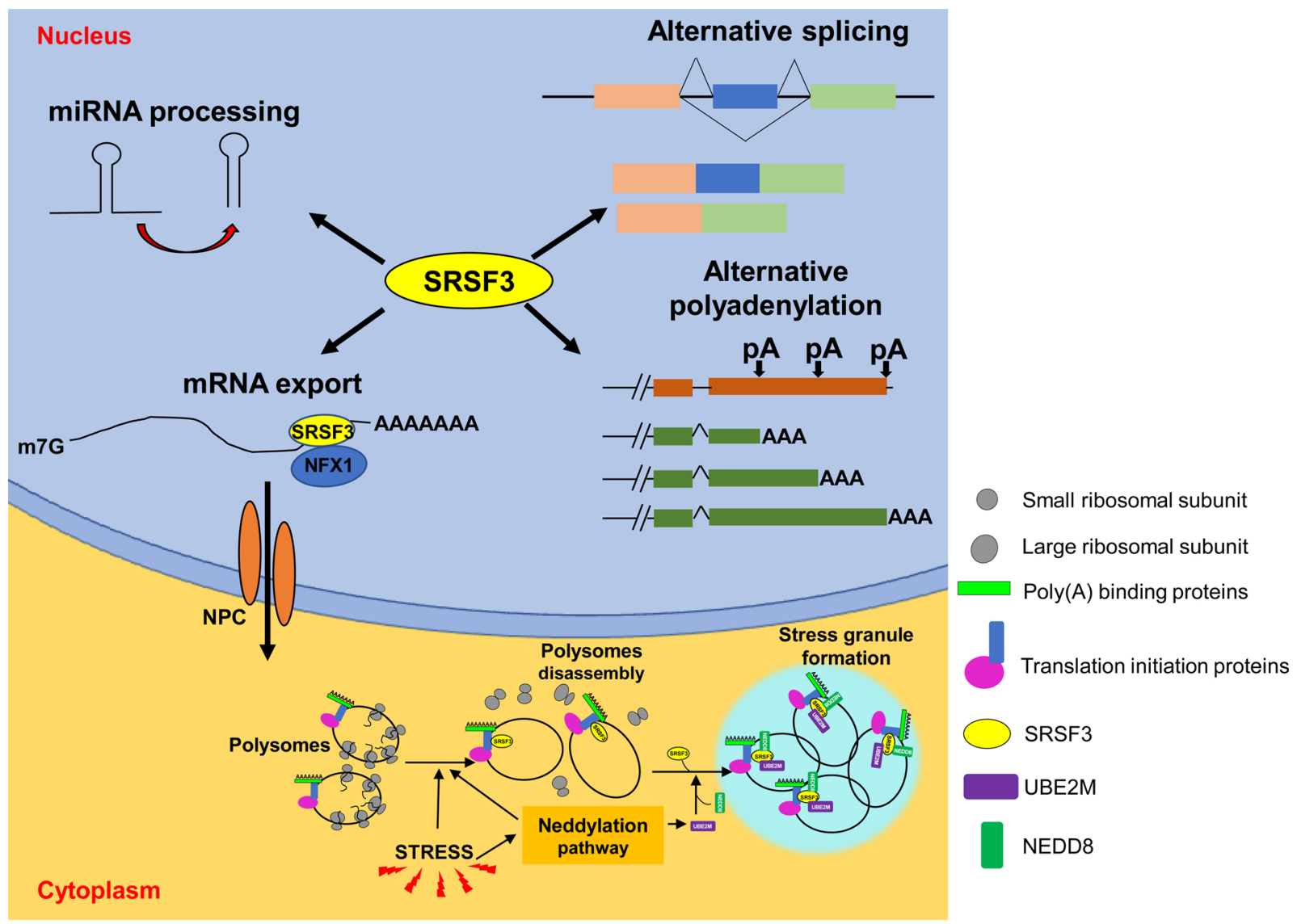

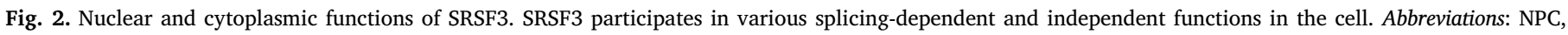
nuclear pore complex; pA, poly adenylation site; and m7G, N 7-methylguanosine.

\section{Role in transcription termination}

Termination of transcription by RNA polymerase II is a complex process and is coupled to 3' end processing machinery (Richard and Manley, 2009). Cui et al. (2008) showed that SRSF3 is involved in events following cleavage of 3'end, leading to transcription termination. They proposed that SRSF3 facilitates transcription termination either by degrading RNA downstream of the cleavage site or by releasing the polymerase from the DNA (Cui et al., 2008).

\section{Role in miRNA biogenesis}

The role of SRSF3 in miRNA biogenesis was first reported by Auyeung et al. (2013). They observed the presence of SRSF3 binding motif (CNNC) downstream of most pri-miRNA hairpins in bilaterian animals. This binding motif was found to be one of the three primarysequence determinants which distinguish pri-miRNAs from other hairpin-containing transcripts, thus facilitating pri-miRNA processing (Auyeung et al., 2013). The importance of SRSF3 in pri-miRNA processing was further strengthened by studies of Chronic lymphocytic leukemia (CLL) patients (Calin et al., 2002, 2005). CLL patients frequently show deletions spanning the intron containing tumor suppressor miRNAs, namely miR-15a and miR-16-1. However, Calin et al. (2002, 2005) identified a loss of heterozygosity for C $>$ T single nucleotide polymorphism (SNP) downstream of the miR-16-1 hairpin in tumors as the cause for miR-16-1 downregulation in 2/75 CLL patients that they studied. This SNP corresponds to the first C in the CNNC motif and disrupts SRSF3 recruitment, thereby affecting miR-16-1 pri-miRNA processing leading to its downregulation. Recently, Kim et al. (2018) demonstrated that the binding of SRSF3 to the CNNC motif regulates pri-miRNA processing by directly recruiting DROSHA to the basal junction in a CNNC-dependent manner, thus enhancing Microprocessor activity. A rare genetic variation found in the conserved terminal loop of pri-miR-30c-1 (G > A) was identified as the cause of increased levels of the mature miRNA in breast and gastric cancer patients (Shen et al., 2009; Hogg et al., 2014). Fernandez et al. (2017) reported that this genetic variation $(\mathrm{G}>\mathrm{A}$ ) leads to secondary structure rearrangement that in turn facilitates binding of SRSF3, resulting in increased levels of miR-30c.

\section{Role in pluripotency}

SRSF3 plays a critical role in development as SRSF3-null mice fail to form blastocysts (Jumaa et al., 1999). Recently, SRSF3 was shown to regulate gene expression during reprogramming stem-cell renewal and early development (Ratnadiwakara et al., 2018). Using a reprogrammable tamoxifen inducible Srsf3 knockout mouse model, Ratnadiwakara et al. (2018) showed that SRSF3 facilitates reprogramming and is essential for maintenance of pluripotency. Apart from regulating the nucleocytoplasmic export of NANOG, SRSF3 coordinates the pluripotency gene expression program by binding to multiple other (pre-)mRNAs encoding core pluripotency transcription factors, chromatin modifiers, RNA processing factors including the export factor Nfx1 (Ratnadiwakara et al., 2018).

\section{Role in DNA repair}

The role of SRSF3 as a novel regulator of homologous recombination-mediated DNA repair (HRR) pathway especially in the context of neoplastic transformation was recently established by He and Zhang (2015). They showed that knockdown of SRSF3 leads to downregulation of key genes involved in the HRR pathway, namely breast 
cancer 1, early onset (BRCA1), BRCA1 interacting protein C-terminal helicase1 (BRIP1), and RAD51 recombinase (RAD51) through an epigenetic pathway (He and Zhang, 2015). During transcription, hybridization between the template DNA and the nascent RNA can lead to generation of RNA:DNA hybrid structures called as R-loops which are deleterious for the cells as they induce DNA double strand breaks and large scale DNA rearrangements that can trigger genome instability. Using chicken B cell line DT40, Li and Manley (2005) observed that SRSF1 gets co-transcriptionally deposited on the nascent RNA from the RNA polymerase II C-terminal domain and suppresses R-loop formation and promotes messenger ribonucleoprotein particle (mRNP) assembly. SRSF3 was also reported to suppress R-loop formation during in vitro transcription but was less effective compared to SRSF1 (Li and Manley, 2005). Jim'enez et al. (2019) have recently reported the involvement of truncated SRSF3 in genome instability, which is discussed in detail later in the review. Based on these studies, the potential role of SRSF3 in DNA repair and maintaining genome stability needs to be further explored.

\section{Role in quality control of nuclear RNA}

The process of mRNA processing and formation of an mRNP competent for export into the cytoplasm is an error-prone process. Aberrantly processed mRNPs that fail the quality control steps of the surveillance mechanism are retained in the nucleus and are degraded by various ribonucleases (Eberle and Visa, 2014). RNA exosome pathway is one of the two major pathways involved in degradation of defective mRNA transcripts. Using Epstein Barr virus mRNA as a model, Mure et al. (2018) proposed a novel model for coupling between splicing machinery and RNA exosome mediated mRNA decay machinery. They demonstrated that in the absence of the viral EB2 protein, SRSF3 loaded onto the newly synthesized viral mRNAs expressed from intronless genes recruits the splicing machinery and the NEXT complex together with the RNA exosome (Mure et al., 2018). This leads to destabilization of the mRNAs and their subsequent decay. However, when EB2 is present, it interacts with SRSF3 and prevents the nuclear RNA exosome's access to the mRNAs and thus prevents their decay, suggesting that SRSF3 aids the RNA exosome and the NEXT complex in the recognition and decay of certain mRNAs (Mure et al., 2018).

\section{Role in stress granule assembly}

SRSF3 was identified as a potential regulator as well as major structural component of translationally stalled mRNA-protein complexes, namely the stress granule (SG) and P-body (PB) (Yoon et al., 2013). Jayabalan et al. (2016) have further studied the effect of arsenite-induced oxidative stress on SG assembly. They observe that, in response to arsenite, SRSF3 is selectively neddylated at Lys8, which in turn induces SG assembly, emphasizing the importance of neddylation pathway in SG assembly (Jayabalan et al., 2016).

\section{Role in maintaining transcriptional integrity of oocytes}

Jumaa et al. (1999) have shown that SRSF3 is present throughout various embryonic stages like oocytes, fertilized egg, 8-cell embryo and blastocyst indicating that maternal SRSF3 is packaged into the developing egg. The role of maternal SRSF3 during oocyte maturation was recently established by Do et al. (2018). Using maternal Srsf3-knockout oocytes, they established that depletion of maternal SRSF3 protein results in developmental arrest at one/two-cell stage, indicating that maternal SRSF3 is essential for preimplantation development (Do et al., 2018). Further, during the development of fertilization-competent oocytes, maternal SRSF3 maintains transcriptome integrity of the developing oocyte by acting as an essential regulator of alternative splicing and of transposable elements (Do et al., 2018).

\section{Involvement of SRSF3 in human health and diseases}

\subsection{Role in cancer}

The role of SRSF3 as a proto-oncogene crucial for cell proliferation and tumor induction and maintenance was established by Jia and colleagues (2010). SRSF3 was found to be overexpressed in cancers of the lung, cervix, breast, skin, stomach, bladder, colon, liver, thyroid and kidney, as well as in various mesenchymal tissue-derived tumors (Jia et al., 2010). The significance of chromosome $6 \mathrm{p}$ amplification in progression of several cancers is reviewed by Santos et al. (2007). The SRSF3 gene is also located on chromosome 6p and, Jia et al. (2010) showed that the gene amplification was indeed the cause for increased SRSF3 expression in a set of lung cancer and cervical cancer samples. SRSF3 was also found to be overexpressed in ovarian cancer patients and required for tumor cell growth and survival (He et al., 2011). Expression of SRSF3 was found to be upregulated and correlated with carcinogenesis and progression of oral squamous cell carcinoma (Peiqi et al., 2016). SRSF3 was identified as a direct target of the oncogenic Wnt/ß-catenin/TCF4 signalling pathway and a direct correlation was found between activated Wnt signaling and SRSF3 expression in colon cancer stem cells (Goncalves et al., 2008; Corbo et al., 2012). Recently, SRSF3 was shown to regulate the expression of miR-1908-5p by activating the NF- $\mathrm{kB}$ pathway (Kim et al., 2017a). In turn, miR-1908-5pmediated downregulation of NF- $\mathrm{KB}$ inhibitor interacting Ras-like 2 (NKIRAS2), a negative regulator of the NF- $\mathrm{kB}$ pathway, was attributed to the oncogenic functions of SRSF3 (Kim et al., 2017a). Knockdown of SRSF3 in human osteosarcoma U2OS cells was found to regulate the expression of at least 20 miRNAs, including a subset of tumor suppressor and oncogenic miRNAs (Ajiro et al., 2015). Table 3 contains a list of SRSF3-regulated miRNAs that are potentially involved in cancer pathogenesis. SRSF3-mediated tumorigenesis is also promoted through its regulation of alternative splicing of key target genes like pyruvate kinase M (PK-M), involved in aerobic glycolysis and tumor suppressor gene programmed cell death 4 (PDCD4) (Wang et al., 2012; Park and Jeong, 2016). Apart from its proto-oncogenic role, SRSF3 has also been shown to act as tumor suppressor in liver and colorectal cancers (Sen et al., 2015; Kumar et al., 2019; Torres et al., 2018). Using a hepatocyte-specific SRSF3 knockout mice, Sen et al. (2015) have shown that SRSF3 plays a protective role in preventing hepatic carcinogenesis by regulating splicing to suppress steatosis, fibrosis, mitogenesis and EMT in hepatocytes. SRSF3 was also found to be either decreased or mislocalized in the nucleus in hepatocellular carcinoma patient samples (Sen et al., 2015). Kumar et al. (2019) have recently shown that palmitic acid-induced oxidative stress leads to selective neddylation of SRSF3 at lysine 11 which targets SRSF3 for proteasome mediated degradation. They speculate that this neddylation-dependent regulation of SRSF3 might be disrupted in early metabolic liver diseases and may contribute to the progression to non-alcoholic steatohepatitis (NASH), cirrhosis and eventually hepatocellular carcinoma (Kumar et al., 2019). The tumor suppressor role of SRSF3 has also been seen in case of colorectal cancer by Torres et al. (2018) who observed that there is a gradual loss of SRSF3 expression which associated with cancer progression and correlated significantly with poor survival and shorter disease-free survival.

\subsection{Role in senescence}

SRSF3 was found to regulate the p53-mediated cellular senescence pathway through alternative splicing of TP53 mRNA (Tang et al., 2013). It is downregulated in replicatively senescent cells, leading to TP53 mRNA splicing towards the p53 $\beta$ isoform, which in turn induces cellular senescence (Tang et al., 2013). The authors also speculate that inactivation of E2F, a transcription factor which transactivates SRSF3 expression and is frequently inactivated during senescence (Jumaa et al., 1997; Narita et al., 2003; Lazzerini et al., 2005; Lanigan et al., 
2011), results in SRSF3 downregulation. Recently, Shen et al. (2019) found another mechanistic explanation for SRSF3 downregulation-induced cellular senescence. They showed that knockdown of SRSF3 results in preferential usage of proximal ploy(A) sites and thus global shortening of the 3'UTRs of mRNAs with a specific enrichment of genes involved in senescent-associated pathways (Shen et al., 2019). These shortened 3'UTR mRNAs produce more protein compared to the longer ones (Shen et al., 2019). SRSF3 downregulation thus modulates polyadenylation and induces cellular senescence by increasing the production of senescent associated genes (Shen et al., 2019).

\subsection{Role in neurological disorders}

Several neurological and psychiatric disorders have been reported to be associated with defects in alternative splicing (Lee and Irizarry, 2003; Garcia-Blanco et al., 2004). Tau proteins are microtubule-associated proteins that function in microtubule (MT) assembly and stabilization via their carboxyl-terminal tandem repeat sequences. The human TAU gene, located on chromosome 17q21, consists of 16 exons and the alternative splicing of three exons, namely 2,3 and 10 gives rise to six different isoforms of tau protein (Goedert and Jakes, 1990; Lee et al., 2001). These isoforms differ by the presence of either three (3Rtau) or four (4R-tau) carboxyl-terminal tandem repeat sequences of 31 or 32 amino acids (Goedert and Jakes, 1990; Lee et al., 2001). Alternative splicing of exon 10 produces the 3R-tau or 4R-tau (Lee et al., 2001). Compared to the 3R-tau isoforms, the 4R-tau isoforms have greater MT binding affinity and are also more efficient at promoting MT polymerization (Goedert and Jakes, 1990; Yu et al., 2004). In adult human brain, the ratio of 3R-tau to 4R-tau is $\sim 1$. Apart from the major class of mutations in the coding sequences which affect the ability of tau to interact with MTs and promote MT assembly, other mutations which affect the alternative splicing of exon 10 and thereby skew the $3 R / 4 R$ tau ratios in favour of the 4R tau have been identified in patients suffering from the neurogenerative disorder called familial frontotemporal dementia and parkinsonism linked to chromosome-17 (FTDP17) ( $Y u$ et al., 2004). Yu et al. (2004) identified SRSF3 as a critical regulator of tau exon 10 splicing. They observed that SRSF3 enables exon 10 exclusion in a dose-dependent manner and could also induce skipping of exon 10 from pre-mRNAs containing mutations identified in FTDP-17 patients (Yu et al., 2004). Earlier studies have also described the involvement of other splicing factors like SRSF4, SRSF6 and Tra2 $\beta$ in regulation of tau exon 10 splicing (Gao et al., 2000; Jiang et al., 2003). Abundant filamentous inclusions of tau seen in FTDP-17 patients are also the pathological characteristics of many other neurodegenerative tauopathies, such as Alzheimer's disease, Pick disease, progressive supranuclear palsy and corticobasal degeneration (Goedert and Spillantini, 2000). Therefore, identification of compounds that can modulate tau splicing has promising therapeutic potential for many of these neurodegenerative tauopathies. SRSF3 was also found to regulate the alternative splicing of tropomyosin receptor kinase B (TrkB) gene (Wong et al., 2012). In Alzheimer's disease, elevated level of SRSF3 selectively increases the TrkB-Shc transcript which interferes with the $\mathrm{BDNF} / \mathrm{TrkB}-\mathrm{TK}+$ signalling in neurons and contributes to the disease pathology (Wong et al., 2012). SRSF3 was also reported to be increased at mRNA levels in bipolar disorder patients (Watanuki et al., 2008).

\subsection{Role in cardiac diseases}

A recent study by Ortiz-Sánchez et al. (2019) showed that SRSF3 is vital for heart development and homeostasis using a mouse model. Cardiac SRSF3 expression was found to be highest at mid-gestation and gradually decreases during later embryonic stages and the postnatal period (Ortiz-Sánchez et al., 2019). Using cardiac-specific knockout mouse model, SRSF3 was shown to be essential for cell proliferation, proper heart development, and subsequent embryonic survival (OrtizSánchez et al., 2019). Also, cardiac-specific knockout in adult mice

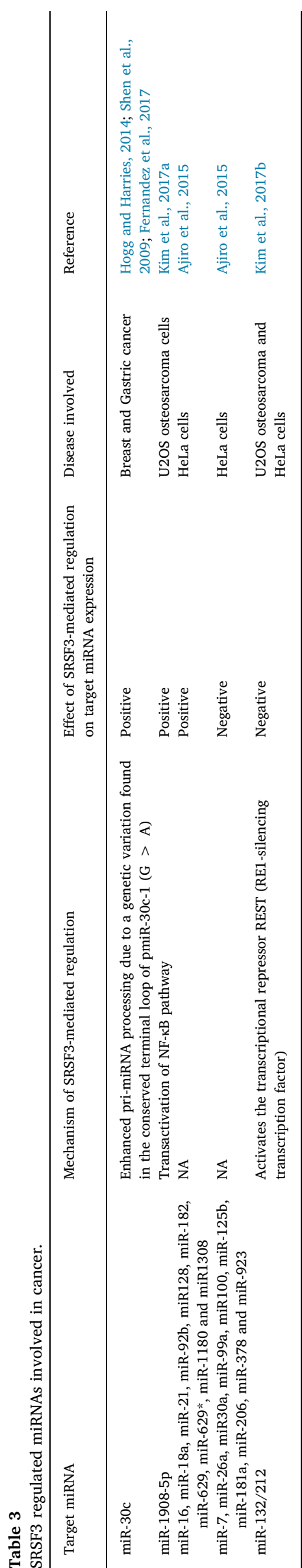


heart was found to lead to premature death due to severe cardiac contraction defects that lead to heart failure (Ortiz-Sánchez et al., 2019). Loss of SRSF3, which leads to decapping and degradation of mRNAs specifically those that encode sarcomeric proteins, was reported as the foremost mechanism for the observed cardiac dysfunction. Apart from its role in cardiac development and homeostasis, SRSF3 was also shown to be dysregulated during myocardial infarction (MI) (OrtizSánchez et al., 2019). Following MI, SRSF3 expression decreases at both mRNA and protein levels along with SRSF1 with an increase in the exon-4 included SRSF3 (Ortiz-Sánchez et al., 2019). The decreased SRSF1 level is thought to favour inclusion of SRSF3 exon 4, leading to SRSF3 downregulation after MI (Ortiz-Sánchez et al., 2019).

\section{Autoregulation of SRSF3}

SRSF3 is involved in regulation of alternative splicing of its own mRNA and autoregulates the expression to maintain homeostasis (Jumaa and Nielsen, 1997). This autoregulation of SRSF3 was first reported by Jumaa and Nielsen (1997) who showed that overexpression of SRSF3 results in the reduction in the level of exon 4-skipped transcript variant-1 and activates the production of transcript variant-2 which includes exon 4. Jumaa et al. (1997) also showed that expression of the exon 4-included transcript increases compared to the exon 4skipped transcript in starved (G0) cells. However, as soon as the starved cells are serum stimulated, there is a loss of the exon 4-included form and induction of the exon 4-skipped form (Jumaa et al., 1997). SRSF3 activates exon 4 use, while other SR proteins like SRSF1 and SRSF2 inhibit its inclusion and Tra2, SRSF5 and SRSF7 show no effect on exon 4 usage (Jumaa and Nielsen, 2000). Autoregulation thus plays an important role in maintaining the steady state levels of SRSF3. There is growing evidence to show that SRSF3 autoregulation is disrupted in cancers and plays a critical role in the process of carcinogenesis. In oral squamous cell carcinoma (OSCC) cells, it was found that Polypyrimidine Tract Binding Protein 1 (PTBP1) and Polypyrimidine Tract Binding Protein 2 (PTBP2) impair SRSF3 autoregulation, leading to overexpression of SRSF3 protein by binding to an exonic splicing suppressor in exon 4 and preventing its inclusion (Guo et al., 2015). Heterogeneous nuclear ribonucleoprotein L (HnRNP L) was also shown to regulate the transcriptional and alternative splicing of SRSF3 (Jia et al., 2016). CircSMARCA5, a tumor suppressor circular RNA involved in glioblastoma multiforme (GBM) pathogenesis, tethers the oncoprotein SRSF1 and therefore indirectly regulates SRSF3 by inducing the expression of exon 4-included transcript (Barbagallo et al., 2018).

\section{Newly identified independent functions of truncated SRSF3}

Recent reports have attributed independent biological functions to the transcript variant-2 encoded truncated SRSF3 protein (SRSF3-TR), specifically in cancer cells. Kano et al. (2014) reported that on exposure of HCT116 colon cancer cells to an oxidant, namely sodium arsenite, there is an increase in the expression of SRSF3-TR which in turn positively regulates oxidative stress-stimulated interleukin- 8 production through regulation of c-Jun production. SRSF3-TR might therefore positively regulate oxidative stress-initiated inflammatory response in colon cancer cells (Kano et al., 2014). The splicing regulator SLU7, which protects cells from transcription-associated genome instability, is found to be downregulated while SRSF3-TR expression is elevated in the liver of the patients with cirrhosis and hepatocellular carcinoma (Castillo et al., 2009; Jim'enez et al., 2019). SRSF3-TR impairs the correct splicing and expression of SRSF1 and the critical sister chromatid cohesion protein soronin (Jim'enez et al., 2019). SLU7 thus prevents genome instability by regulating SRSF3 splicing and through the expression of miR-17 (Jim'enez et al., 2019). Further, SLU7 regulates SRSF1 and SRSF3 splicing and prevents the expression of SRSF3TR (Jim'enez et al., 2019). SLU7 also regulates miR-17 expression which in turn targets SRSF3-TR for degradation (Jim'enez et al., 2019).
Contrarily, Chang et al. (2018) reported that Amiodarone, a class III antiarrhythmic agent, promotes cancer cell death through elevated truncated SRSF3.

\section{Concluding remarks}

Apart from their role as modulators of constitutive and alternative splicing, many splicing factors including SRSF3 control other aspects of RNA metabolism and gene regulation. Alterations in their expression thus has far reaching consequences for the cell functioning. The implication of deregulated splicing factors expression in various diseases including cancers has now been widely established. Modulation of splicing factors is therefore a potential therapeutic approach for targeting a variety of human diseases. Recent studies have focused on identifying novel or repurposed splicing factor modulators and exploring their therapeutic potential. For example, cardiotonic steroids like Digitoxin and Digoxin have emerged as efficient regulators of alternative splicing through their effect on SRSF3 and Tra2- $\beta$ expression (Anderson et al., 2012; Wong et al., 2013). However, a recent report by Chung et al. (2017) on the long-term usage of Digoxin in heart failure patients and an associated risk of developing cancer in these patients calls attention to the safety of these splicing modulators in the long run. Modulating the splicing profile in disease conditions by employing antisense-oligonucleotides is another exciting therapeutic avenue which should be explored further (Dewaele et al., 2016). Sun et al. (2019) recently reported that antisense-oligonucleotides mediated downregulation of SRSF3 sensitized OSCC and breast cancer cells to the chemotherapeutic drug Paclitaxel. This study serves as a proof of concept and further underscores the need to develop treatment modalities against SRSF3.

\section{Source of funding}

Financially support in AK laboratory from DBT (Grant\# BT/ PR10272/BRB/10/1266/2013 and BT/PR8670/AGR/36/757/2013), DST-FIST [SR/FST/LS11-036/2014(C)], UGC-SAP [F.4.13/2018/DRSIII (SAP-II)] and DBT-IISc Partnership Program Phase-II (BT/PR27952INF/22/212/2018) is gratefully acknowledged.

\section{Declaration of Competing Interest}

Both authors declare that there are no conflicts of interests, no financial affiliation or involvement with any commercial organization with direct financial interest in the subject or materials discussed in this manuscript.

\section{References}

Ajiro, M., Jia, R., Yang, Y., Zhu, J., Zheng, Z.M., 2015. A genome landscape of SRSF3regulated splicing events and gene expression in human osteosarcoma U2OS cells. Nucleic Acids Res. 44, 1854-1870.

Anderson, E.S., Lin, C.H., Xiao, X., Stoilov, P., Burge, C.B., Black, D.L., 2012. The cardiotonic steroid digitoxin regulates alternative splicing through depletion of the splicing factors SRSF3 and TRA2B. RNA 18, 1041-1049.

Aubol, B.E., Plocinik, R.M., Hagopian, J.C., Ma, C.T., McGlone, M.L., Bandyopadhyay, R., Fu, X.D., Adams, J.A., 2013. Partitioning RS domain phosphorylation in an SR protein through the CLK and SRPK protein kinases. J. Mol. Biol. 425, 2894-2909.

Auyeung, V.C., Ulitsky, I., McGeary, S.E., Bartel, D.P., 2013. Beyond secondary structure: primary-sequence determinants license pri-miRNA hairpins for processing. Cell 152, 844-858.

Barbagallo, D., Caponnett, A., Cirnigliaro, M., Brex, D., Barbagallo, C., D’Angeli, F., Morrone, A., Caltabiano, R., Barbagallo, G.M., Ragusa, M., Di Pietro, C., Hansen, T.B., Purrello, M., 2018. CircSMARCA5 inhibits migration of glioblastoma multiforme cells by regulating a molecular axis involving splicing factors SRSF1/SRSF3/PTB. Int. J. Mol. Sci. 19 (2). https://doi.org/10.3390/ijms19020480. pii: E480.

Buoso, E., Ronfani, M., Galasso, M., Ventura, D., Corsini, E., Racchi, M., 2019. Cortisolinduced SRSF3 expression promotes GR splicing, RACK1 expression and breast cancer cells migration. Pharmacol. Res. 143, 17-26.

Cá ceres, J.F., Misteli, T., Screaton, G.R., Spector, D.L., Krainer, A.R., 1997. Role of the modular domains of SR Proteins in subnuclear localization and alternative splicing specificity. J. Cell Biol. 138, 225-238. 
Ca'ceres, J.F., Screaton, G.R., Krainer, A.R., 1998. A specific subset of SR proteins shuttles continuously between the nucleus and the cytoplasm. Genes Dev. 12, 55-66.

Calin, G.A., Dumitru, C.D., Shimizu, M., Bichi, R., Zupo, S., Noch, E., Aldler, H., Rattan, S., Keating, M., Rai, K., Rassenti, L., Kipps, T., Negrini, M., Bullrich, F., Croce, C.M., 2002. Frequent deletions and downregulation of micro-RNA genes miR15 and miR16 at $13 q 14$ in chronic lymphocytic leukemia. Proc. Natl. Acad. Sci. U. S. A. 99, 15524-15529.

Calin, G.A., Ferracin, M., Cimmino, A., Di Leva, G., Shimizu, M., Wojcik, S.E., Iorio, M.V., Visone, R., Sever, N.I., Fabbri, M., Iuliano, R., Palumbo, T., Pichiorri, F., Roldo, C., Garzon, R., Sevignani, C., Rassenti, L., Alder, H., Volinia, S., Liu, C., Kipps, T.J., Negrini, M., Croce, C.M., 2005. A microRNA signature associated with prognosis and progression in chronic lymphocytic leukemia. N. Engl. J. Med. 353, 1793-1801.

Castillo, J., Go ni, S., Latasa, M.U., Perugorr’́a, M.J., Calvo, A., Muntan’e, J., Bioulac Sage, P., Balabaud, C., Prieto, J., Avila, M.A., Berasain, C., 2009. Amphiregulin induces the alternative splicing of p73 into its oncogenic isoform DeltaEx2p73 in human hepatocellular tumors. Gastroenterol 137, 1805-1815.

Cavaloc, Y., Bourgeois, C.F., Kister, L., Stevenin, J., 1999. The splicing factors 9 G8 and SRp20 transactivate splicing through different and specific enhancers. RNA 5, 468-483.

Cazalla, D., Zhu, J., Manche, L., Huber, E., Krainer, A.R., Ca'ceres, J.F., 2002. Nuclear export and retention signals in the RS domain of SR proteins. Mol. Cell. Biol. 22, 6871-6882.

Chang, Y.L., Liu, S.T., Wang, Y.W., Lin, W.S., Huang, S.M., 2018. Amiodarone promotes cancer cell death through elevated truncated SRSF3 and downregulation of miR-224. Oncotarget 9, 13390-13406.

Chung, M.H., Wang, Y.W., Chang, Y.L., Huang, S.M., Lin, W.S., 2017. Risk of cancer in patients with heart failure who use digoxin: a 10-year follow-up study and cell-based verification. Oncotarget 8, 44203-44216.

Colwill, K., Pawson, T., Andrews, B., Prasad, J., Manley, J.L., Bell, J.C., Duncan, P.I., 1996. The Clk/Sty protein kinase phosphorylates SR splicing factors and regulates their intranuclear distribution. EMBO J. 15, 265-275.

Corbo, C., Orru’, S., Gemei, M., Noto, R.D., Mirabelli, P., Imperlini, E., Ruoppolo, M., Vecchio, L.D., Salvatore, F., 2012. Protein cross- talk in CD133+ colon cancer cells indicates activation of the Wnt pathway and upregulation of SRp20 that is potentially involved in tumorigenicity. Proteomics 12, 2045-2059.

Corkery, D.P., Holly, A.C., Lahsaee, S., Dellaire, G., 2015. Connecting the speckles: splicing kinases and their role in tumorigenesis and treatment response. Nucleus 6, 279-288.

Cowper, A.E., Ca'ceres, J.F., Mayeda, A., Screaton, G.R., 2001. Serine-Arginine (SR) protein-like factors that antagonize authentic SR proteins and regulate alternative splicing. J. Biol. Chem. 276, 48908-48914.

Cui, M., Allen, M.A., Larsena, A., MacMorris, M., Hana, M., Blumenthala, T., 2008. Genes involved in pre-mRNA 3'-end formation and transcription termination revealed by a lin-15 operon Muv suppressor screen. Proc. Natl. Acad. Sci. U. S. A. 105, 16665-16670.

Cullen, B.R., 2000. Nuclear RNA export pathways. Mol. Cell. Biol. 20 (12), 4181-4187.

Derti, A., Garrett-Engele, P., MacIsaac, K.D., Stevens, R.C., Sriram, S., Chen, R., Rohl, C.A., Johnson, J.M., Babak, T., 2012. A quantitative atlas of polyadenylation in five mammals. Genome Res. 22, 1173-1183.

Dewaele, M., Tabaglio, T., Willekens, K., Bezzi, M., Teo, S.X., Low, D.H.P., Koh, C.M., Rambow, F., Fiers, M., Rogiers, A., Radaelli, E., Al-Haddawi, M., Tan, S.Y., Hermans, E., Amant, F., Yan, H., Lakshmanan, M., Koumar, R.C., Lim, S.T., Derheimer, F.A., Campbell, R.M., Bonday, Z., Tergaonkar, V., Shackleton, M., Blattner, C., Marine, J.C., Guccione, E., 2016. Antisense oligonucleotide- mediated MDM4 exon 6 skipping impairs tumor growth. J. Clin. Invest. 126, 68-84.

Do, D.V., Strauss, B., Cukuroglu, E., Macaulay, I., Wee, K.B., Hu, T.X., Mozos, I., Lee, C., Harrison, A., Butler, R., Dietmann, S., Jernej, U., Marioni, J., Smith, C.W.J., Göke, J., Surani, M.A., 2018. SRSF3 maintains transcriptome integrity in oocytes by regulation of alternative splicing and transposable elements. Cell Discov. 4, 33.

Eberle, A.B., Visa, N., 2014. Quality control of mRNP biogenesis: networking at the transcription site. Sem. Cell Dev. Biol. 32, 37-46.

Fernandez, N., Cordiner, R.A., Young, R.S., Hug, N., Macias, S., Ca'ceres, J.F., 2017. Genetic variation and RNA structure regulate microRNA biogenesis. Nat. Commun. 8, 15114.

Galiana-Arnoux, D., Lejeune, F., Gesnel, M.C., Stevenin, J., Breathnach, R., GattoKonczak, F.D., 2003. The CD44 alternative v9 exon contains a splicing enhancer responsive to the SR proteins 9G8, ASF/SF2, and SRp20. J. Biol. Chem. 278, 32943-32953.

Gao, Q.S., Memmott, J., Lafyatis, R., Stamm, S., Screaton, G., Andreadis, A., 2000. Complex regulation of tau exon 10 , whose missplicing causes frontotemporal dementia. J. Neurochem. 74, 490-500.

Garcia-Blanco, M.A., Baraniak, A.P., Lasda, E.L., 2004. Alternative splicing in disease and therapy. Nat. Biotechnol. 22, 535-546.

Goedert, M., Jakes, R., 1990. Expression of separate isoforms of human tau protein: correlation with the tau pattern in brain and effects on tubulin polymerization. EMBO J. 9, 4225-4230.

Goedert, M., Spillantini, M.G., 2000. Tau mutations in frontotemporal dementia FTDP-17 and their relevance for Alzheimer's disease. Biochim. Biophys. Acta 1502, 110-121.

Goncalves, V., Matos, P., Jordan, P., 2008. The b-catenin/TCF4 pathway modifies alternative splicing through modulation of SRp20 expression. RNA 14, 2538-2549.

Goncalves, V., Matos, P., Jordan, P., 2009. Antagonistic SR proteins regulate alternative splicing of tumor-related Rac1b downstream of the PI3-kinase and Wnt pathways. Human Mol. Genet. 18, 3696-3707.

Gruber, A.J., Zavolan, M., 2019. Alternative cleavage and polyadenylation in health and disease. Nat. Rev. Genet. 20, 599-614.

Guo, J., Jia, J., Jia, R., 2015. PTBP1 and PTBP2 impaired autoregulation of SRSF3 in cancer cells. Scientific Rep. 5, 14548.

Hautbergue, G.M., Hung, M.L., Golovanov, A.P., Lian, L.Y., Wilson, S.A., 2008, Mutually exclusive interactions drive handover of mRNA from export adaptors to TAP. Proc. Natl. Acad. Sci. U. S. A. 105, 5154-5159.

He, X., Ee, P.L.R., Coon, J.S., Beck, W.T., 2004. Alternative splicing of the multidrug resistance protein 1/ATP binding cassette transporter subfamily gene in ovarian cancer creates functional splice variants and is associated with increased expression of the splicing factors PTB and SRp20. Clin. Cancer Res. 10, 4652-4660.

He, X., Arslan, A.D., Pool, M.D., Ho, T.T., Darcy, K.M., Coon, J.S., Beck, W.T., 2011. Knockdown of splicing factor SRp20 causes apoptosis in ovarian cancer cells and its expression is associated with malignancy of epithelial ovarian cancer. Oncogene 30, 356-365.

He, X., Zhang, P., 2015. Serine/arginine-rich splicing factor 3 (SRSF3) regulates homologous recombination mediated DNA repair. Mol. Cancer 14, 158.

Hogg, D.R., Harries, L.W., 2014. Human genetic variation and its effect on miRNA biogenesis, activity and function. Biochem. Soc. Trans. 42, 1184-1189.

Huang, Y., Steitz, J.A., 2001. Splicing Factors SRp20 and 9G8 promote the nucleocytoplasmic export of mRNA. Mol. Cell 7, 899-905.

Jayabalan, A.K., Sanchez, A., Park, R.Y., Yoon, S.P., Kang, G.Y., Baek, J.H., Anderson, P., Kee, Y., Ohn, T., 2016. NEDDylation promotes stress granule assembly. Nat. Commun. 7, 12125.

Ji, Z., Lee, J.Y., Pan, Z., Jiang, B., Tian, B., 2009. Progressive lengthening of 3' untranslated regions of mRNAs by alternative polyadenylation during mouse embryonic development. Proc. Natl. Acad. Sci. U. S. A. 106, 7028-7033.

Jia, R., Li, C., McCoy, J.P., Deng, C.X., Zheng, Z.M., 2010. SRp20 is a proto-oncogene critical for cell proliferation and tumor induction and maintenance. Int. J. Biol. Sci. 6, 806-826.

Jia, R., Zhang, S., Liu, M., Zhang, Y., Liu, Y., Fan, M., Guo, J., 2016. HnRNP L is important for the expression of oncogene SRSF3 and oncogenic potential of oral squamous cell carcinoma cells. Scientific Rep. 6, 35976.

Jiang, Z., Tang, H., Havlioglu, N., Zhang, X., Stamm, S., Yan, R., Wu, J.Y., 2003. Mutations in tau gene exon 10 associated with FTDP-17 alter the activity of an exonic splicing enhancer to interact with Tra2 beta. J. Biol. Chem. 278, 18997-19007.

Jim’enez, M., Urtasun, R., Elizalde, M., Azkona, M., Latasa, M.U., Uriarte, I., Arechederra1, M., Alignani, D., B’arcena-Varela, M., A' lvarez-Sola, G., Colyn, L., Santamar'1a, E., Sangro, B., Rodriguez-Ortigosa, C., Ferna'ndez-Barrena, M.G., A' vila, M.A., Berasain, C., 2019. Splicing events in the control of genome integrity: role of SLU7 and truncated SRSF3 proteins. Nucleic Acids Res. 47, 3450-3466.

Jumaa, H., Nielsen, P.J., 1997. The splicing factor SRp20 modifies splicing of its own mRNA and ASF/SF2 antagonizes this regulation. EMBO J. 16, 5077-5085.

Jumaa, H., Nielsen, P.J., 2000. Regulation of SRp20 exon 4 splicing. Biochim. Biophys. Acta 1494, 137-143.

Jumaa, H., Gue'net, J.L., Nielsen, P.J., 1997. Regulated Expression and RNA Processing of Transcripts from the Srp20 splicing factor gene during the cell cycle. Mol. Cell. Biol. 17, 3116-3124.

Jumaa, H., Wei, G., Nielsen, P.J., 1999. Blastocyst formation is blocked in mouse embryos lacking the splicing factor SRp20. Curr. Biol. 9, 899-902.

Kano, S., Nishida, K., Kurebe, H., Nishiyama, C., Kita, K., Akaike, Y., Kajita, K., Kurokawa, K., Masuda, K., Kuwano, Y., Tanahashi, T., Rokutan, K., 2014. Oxidative stress-inducible truncated serine/arginine-rich splicing factor 3 regulates interleukin-8 production in human colon cancer cells. Am. J. Physiol., Cell Physiol. 306, 250-262.

Kasowitz, S.D., Ma, J., Anderson, S.J., Leu, N.A., Xu, Y., Gregory, B.D., Schultz, R.M., Wang, P.J., 2018. Nuclear $\mathrm{m}^{6} \mathrm{~A}$ reader YTHDC1 regulates alternative polyadenylation and splicing during mouse oocyte development. PLoS Genet. 14, e1007412.

Kim, H.R., Shin, C.H., Lee, H., Choi, K.H., Nam, D.H., Ohn, T., Kim, H.H., 2017a. MicroRNA-1908-5p contributes to the oncogenic function of the splicing factor SRSF3. Oncotarget 8, 8342-8355.

Kim, H.R., Hwang, S.J., Shin, C.H., Choi, K.H., Ohn, T., Kim, H.H., 2017b. SRSF3-regulated miR-132/212 controls cell migration and invasion by targeting YAP1. Experimental Cell Res. 358, 161-170.

Kim, K., Nguyen, T.D., Li, S., Nguyen, T.A., 2018. SRSF3 recruits DROSHA to the basal junction of primary microRNAs. RNA 24, 892-898.

Koizumi, J., Okamoto, Y., Onogi, H., Mayeda, A., Krainer, A.R., Hagiwara, M., 1999. The subcellular localization of SF2/ASF is regulated by direct interaction with SR protein kinases (SRPKs). J. Biol. Chem. 274, 11125-11131.

Krainer, A.R., Conway, G.C., Kozak, D., 1990. Purification and characterization of premRNA splicing factor SF2 from HeLa cells. Genes Dev. 4, 1158-1171.

Kumar, D., Das, M., Sauceda, C., Ellies, L.G., Kuo, K., Parwal, P., Kaur, M., Jih, L., Bandyopadhyay, G.K., Burton, D., Loomba, R., Osborn, O., Webster, N.J.G., 2019. Degradation of splicing factor SRSF3 contributes to progressive liver disease. J. Clin. Invest. 4477-4491.

Lackford, B., Yao, C., Charles, G.M., Weng, L., Zheng, X., Choi, E.A., Xie, X., Wan, J., Xing, Y., Freudenberg, J.M., Yang, P., Jothi, R., Hu, G., Shi, Y., 2014. Fip1 regulates mRNA alternative polyadenylation to promote stem cell self-renewal. EMBO J. 33, 878-889.

Lai, M.C., Lin, R.I., Tarn, W.Y., 2001. Transportin-SR2 mediates nuclear import of phosphorylated SR proteins. Proc. Natl. Acad. Sci. U. S. A. 98, 10154-10159.

Lanigan, F., Geraghty, J.G., Bracken, A.P., 2011. Transcriptional regulation of cellular senescence. Oncogene 30, 2901-2911.

Lazzerini, D.E., Attwooll, C., Pasini, D., Helin, K., 2005. Deregulated E2F activity induces hyperplasia and senescence-like features in the mouse pituitary gland. Mol. Cell. Biol. $25,2660-2672$.

Lee, C.J., Irizarry, K., 2003. Alternative splicing in the nervous system: an emerging source of diversity and regulation. Biol. Psychiatry 54, 771-776.

Lee, V., Goedert, M., Trojanowski, J.Q., 2001. Neurodegenerative tauopathies. Annu. Rev. Neurosci. 24, 1121-1159.

Li, X., Manley, J.L., 2005. Inactivation of the SR protein splicing factor ASF/SF2 results in 
genomic instability. Cell 122, 365-378.

Long, Y., Sou, W.H., Yung, K.W.Y., Liu, H., Wan, S.W.C., Li, Q., Zeng, C. Law, C.O.K. Chan, G.H.C., Lau, T.C.K., Ngo, J.C.K., 2019. Distinct mechanisms govern the phosphorylation of different SR protein splicing factors. J. Biol. Chem. 294, 1312-1327.

Lou, H., Neugebauer, K.M., Gagel, R.F., Berget, S.M., 1998. Regulation of alternative polyadenylation by U1 snRNPs and SRp20. Mol. Cell. Biol. 18, 4977-4985.

Manley, J.L., Krainer, A.R., 2010. A rational nomenclature for serine/arginine-rich protein splicing factors (SR proteins). Genes Dev. 24, 1073-1074.

Mata, M., Kornblihtt, A.R., 2006. RNA polymerase II C-terminal domain mediates regulation of alternative splicing by SRp20. Nat. Structural Mol. Biol. 13, 11.

Müller-McNicoll, M., Botti, V., de Jesus Domingues, A.M., Brandl, H., Schwich, O.D., Steiner, M.C., Curk, T., Poser, I., Zarnack, K., Neugebauer, K.M., 2016. SR proteins are NXF1 adaptors that link alternative RNA processing to mRNA export. Genes Dev. 30, 553-566.

Mure, F., Corbin, A., Benbahouche, N., Bertrand, E., Manet, E., Gruffat, H., 2018. The splicing factor SRSF3 is functionally connected to the nuclear RNA exosome for intronless mRNA decay. Scientific Rep. 8, 12901.

Narita, M., Nunez, S., Heard, E., Narita, M., Lin, A.W., Hearn, S.A., Spector, D.L., Hannon, G.J., Lowe, S.W., 2003. Rb-mediated heterochromatin formation and silencing of E2F target genes during cellular senescence. Cell 113, 703-716.

Ortiz-Sánchez, P., Villalba-Orero, M., López-Olañeta, M.M., Larrasa-Alonso, J., SánchezCabo, F., Martí-Gómez, C., Camafeita, E., Gómez-Salinero, J.M., Ramos-Hernández, L., Nielsen, P.J., Vázquez, J., Müller-McNicoll, M., García-Pavía, P., Lara-Pezzi, E., 2019. Loss of SRSF3 in cardiomyocytes leads to decapping of contraction-related mRNAs and severe systolic dysfunction. Circulation Res. 125, 170-183.

Park, S.K., Jeong, S., 2016. SRSF3 represses the expression of PDCD4 protein by coordinated regulation of alternative splicing, export and translation. Biochem. Biophys. Res. Commun. 470, 431-438.

Park, J.E., Yi, H., Kim, Y., Chang, H., Kim, V.N., 2016. Regulation of poly(A) tail and translation during the somatic cell cycle. Mol. Cell 62, 462-471.

Peiqi, L., Zhaozhong, G., Yaotian, Y., Jun, J., Jihua, G., Rong, J., 2016. Expression of SRSF3 is correlated with carcinogenesis and progression of oral squamous cell carcinoma. Int. J. Med. Sci. 13, 533-539.

Ratnadiwakara, M., Archer, S.K., Dent, C.I., Mozos, I., Beilharz, T.H., Knaupp, A.S Nefzger, C.M., Polo, J.M., Anko, M.L., 2018. SRSF3 promotes pluripotency through Nanog mRNA export and coordination of the pluripotency gene expression program. eLife 7, e37419.

Rawcliffe, D.F.R., Osterman, L., Lindsten, H., Holmbergm, M., 2016. The high level of aberrant splicing of ISCU in slow-twitch muscle may involve the splicing factor SRSF3. PLoS One 11, e0165453.

Richard, P., Manley, J.L., 2009. Transcription termination by nuclear RNA polymerases. Genes Dev. 23, 1247-1269.

Santos, G.C., Zielenska, M., Prasad, M., Squire, J.A., 2007. Chromosome 6p amplification and cancer progression. J. Clin. Pathol. 60, 1-7.

Sapra, A.K., Anko, M.L., Grishina, I., Lorenz, M., Pabis, M., Poser, I., Rollins, J., Weiland, E.M., Neugebauer, K.M., 2009. SR protein family members display diverse activities in the formation of nascent and mature mRNPs in vivo. Mol. Cell 34, 179-190.

Schaal, T.D., Maniatis, T., 1999. Selection and characterization of pre-mRNA splicing enhancers: identification of novel SR protein-specific enhancer sequences. Mol. Cell 19, 1705-1719.

Sen, S., Langiewicz, M., Jumaa, H., Nicholas, J.G., 2015. Deletion of splicing factor SRSF3 in hepatocytes predisposes to hepatocellular carcinoma in mice. Hepatology 61, $171-183$.

Shen, J., Ambrosone, C.B., Zhao, H., 2009. Novel genetic variants in microRNA genes and familial breast cancer. Int. J. Cancer 124, 1178-1182

Shen, T., Li, H., Song, Y., Li, L., Lin, J., Wei, G., Ni, T., 2019. Alternative polyadenylation dependent function of splicing factor SRSF3 contributes to cellular senescence. Aging 11, 1356-1388.

Song, X., Wan, X., Huang, T., Zeng, C., Sastry, N., Wu, B., James, C.D., Horbinski, C., Nakano, I., Zhang, W., Hu, B., Cheng, S.Y., 2019. SRSF3-regulated RNA alternative splicing promotes glioblastoma tumorigenicity by affecting multiple cellular processes. Cancer Res. 79, 5288-5301.

Sotillo, E., Barrett, D.M., Black, K.L., Bagashev, A., Oldridge, D., Wu, G., Sussman, R. Lanauze, C., Ruella, M., Gazzara, M.R., Martinez, N.M., Harrington, C.T., Chung, E.Y., Perazzelli, J., Hofmann, T.J., Maude, S.L., Raman, P., Barrera, A., Gill, S., Lacey, S.F., Melenhorst, J.J., Allman, D., Jacoby, E., Fry, T., Mackall, C., Barash, Y., Lynch, K.W., Maris, J.M., Grupp, S.A., Thomas-Tikhonenko, A., 2015. Convergence of acquired mutations and alternative splicing of CD19 enables resistance to CART-19 immunotherapy. Cancer Discov. 5, 1282-1295.

Spector, D.L., Lamond, A.I., 2011. Nuclear speckles. Cold Spring Harb. Perspect. Biol. 3 (2), a000646. https://doi.org/10.1101/cshperspect.a000646.

Sun, Y., Yan, L., Guo, J., Shao, J., Jia, R., 2019. Downregulation of SRSF3 by antisense oligonucleotides sensitizes oral squamous cell carcinoma and breast cancer cells to paclitaxel treatment. Cancer Chemother. Pharmacol. 84, 1133-1143.

Tang, Y., Horikawa, I., Ajiro, M., Robles, A.I., Fujita, K., Mondal, A.M., Stauffer, J.K., Zheng, Z.M., Harris, C.C., 2013. Downregulation of splicing factor SRSF3 induces p53b, an alternatively spliced isoform of p53 that promotes cellular senescence. Oncogene 32, 2792-2798.

Tian, B., Manley, J.L., 2017. Alternative polyadenylation of mRNA precursors. Nat. Rev. Mol. Cell Biol. 18, 18-30.

Torres, S., García-Palmero, I., Marín-Vicente, C., Bartolomé, R.A., Calviño, E., Fernández Aceñero, M.J., Casal, J.I., 2018. Proteomic characterization of transcription and splicing factors associated with a metastatic phenotype in colorectal cancer. J. Proteome Res. 17, 252-264.

Wang, Z., Chatterjee, D., Jeon, H.Y., Akerman, M., Heiden, M.G.V., Cantley, L.C., Krainer, A.R., 2012. Exon-centric regulation of pyruvate kinase $M$ alternative splicing via mutually exclusive exons. J. Mol. Cell Biol. 4, 79-87.

Watanuki, T., Funato, H., Uchida, S., Matsubara, T., Kobayashi, A., Wakabayashi, Y. Otsuki, K., Nishida, A., Watanabe, Y., 2008. Increased expression of splicing factor SRp20 mRNA in bipolar disorder patients. J. Affective Disorders 10, 62-69.

Wong, J., Garner, B., Halliday, G.M., Kwok, J.B.L., 2012. Srp20 regulates TrkB pre-mRNA splicing to generate TrkB-Shc transcripts with implications for Alzheimer's disease. J. Neurochem. 123, 159-171.

Wong, R.W., Balachandran, A., Ostrowski, M.A., Cochrane, A., 2013. Digoxin suppresses HIV-1 replication by altering viral RNA processing. PLoS Pathogen 9, e1003241.

Xiao, W., Adhikari, S., Dahal, U., Chen, Y.S., Hao, Y.J., Sun, B.F., Sun, H.Y., Li, A., Ping, X.L., Lai, W.Y., Wang, X., Ma, H.L., Huang, C.M., Yang, Y., Huang, N., Jiang, G.B., Wang, H.L., Zhou, Q., Wang, X.J., Zhao, Y.L., Yang, Y.G., 2016. Nuclear $\mathrm{m}^{6}$ A reader YTHDC1 regulates mRNA splicing. Mol. Cell 61, 507-519.

Yoon, S.P., Kim, H.H., Kim, J., Park, R.Y., Ohn, T., 2013. Regulation of cellular RNA nanoparticle assembly by splicing factor SRp20. J. Nanosci. Nanotechnol. 13, 184-187.

Yu, Q., Guo, J., Zhou, J., 2004. A minimal length between tau exon 10 and 11 is required for correct splicing of exon 10. J. Neurochem. 90, 164-172.

Zahler, A.M., William, S.L., Stolk, J.A., Roth, M.B., 1992. SR proteins: a conserved family of pre-mRNA splicing factors. Genes Dev. 6, 837-847.

Zhou, Z., Fu, X.D., 2013. Regulation of splicing by SR proteins and SR protein-specific kinases. Chromosoma 122, 191-207. 